\title{
Neurocompensatory Effects of the Default Network in Older Adults
}

\author{
Bryant M. Duda ${ }^{1 *}$, Max M. Owens ${ }^{1}$, Emily S. Hallowell ${ }^{1}$ and Lawrence H. Sweet ${ }^{1,2}$ \\ ${ }^{1}$ Department of Psychology, University of Georgia, Athens, GA, United States, ${ }^{2}$ Department of Psychiatry \& Human \\ Behavior, The Warren Alpert Medical School of Brown University, Providence, RI, United States
}

OPEN ACCESS

Edited by:

Panteleimon Giannakopoulos, Université de Genève, Switzerland

Reviewed by:

Manuela Berlingeri, University of Urbino Carlo Bo, Italy Nichole Lighthall,

University of Central Florida, United States

Lindsay Conner,

University of Central Florida, United States, in collaboration with reviewer NL

*Correspondence: Bryant M. Duda Bduda@uga.edu

Received: 22 April 2018 Accepted: 29 April 2019 Published: 04 June 2019

Citation:

Duda BM, Owens MM Hallowell ES and Sweet LH (2019) Neurocompensatory Effects of the Default Network in Older Adults. Front. Aging Neurosci. 11:111. doi: 10.3389/fnagi.2019.00111
The hemispheric asymmetry reduction in older adults (HAROLD) is a neurocompensatory process that has been observed across several cognitive functions but has not yet been examined in relation to task-induced relative deactivations of the default mode network. The present study investigated the presence of HAROLD effects specific to neural activations and deactivations using a functional magnetic resonance imaging (fMRI) n-back paradigm. It was hypothesized that HAROLD effects would be identified in relative activations and deactivations during the paradigm, and that they would be associated with better 2-back performance. Forty-five older adults $(M$ age $=63.8$; range $=53-83$ ) were administered a verbal $n$-back paradigm during $\mathrm{fMRI}$. For each participant, the volume of brain response was summarized by left and right frontal regions of interest, and laterality indices (LI; i.e., left/right) were calculated to assess HAROLD effects. Group level results indicated that age was significantly and negatively correlated with LI (i.e., reduced left lateralization) for deactivations, but positively correlated with LI (i.e., increased left lateralization) for activations. The relationship between age and $\mathrm{LI}$ for deactivation was significantly moderated by performance level, revealing a stronger relationship between age and $\mathrm{LI}$ at higher levels of 2-back performance. Findings suggest that older adults may employ neurocompensatory processes specific to deactivations, and task-independent processes may be particularly sensitive to age-related neurocompensation.

Keywords: older adults, neurocompensation, fMRI, default mode network, HAROLD

\section{INTRODUCTION}

The number of individuals aged 65 or older is projected to exceed 1.5 billion in 2050 (National Institute on Aging and World Health Organization, 2011) and will comprise approximately $30 \%$ of the population by 2060 (Parker et al., 2012). While this expansion is due in part to advancements in healthcare, the aging population will present new challenges for older adults (OAs) and their caretakers. In addition to OAs affected by neurodegenerative conditions, such as Alzheimer's disease (Alzheimer's Disease International, 2010), OAs without such conditions also exhibit wellstudied patterns of neurocognitive decline. These include declines in processing speed (Salthouse, 1996; Charness, 2008), selective attention (Barr and Giambra, 1990; Madden, 1990), working memory (WM) (Balota et al., 2000; Zacks et al., 2000), and task-switching (Kray and Lindenberger, 2000; Kray et al., 2002). Underscoring their functional significance, these decrements have been linked to declines in independent living among healthy OAs (Suchy et al., 2011; Duda et al., 2014). 
Consistent with cognitive findings, neuroimaging studies have identified patterns of neural changes in non-demented OAs. Structural neuroimaging studies have revealed age-related reductions in prefrontal and parietal white matter integrity (Persson et al., 2005; Grady, 2012) and hippocampal and prefrontal gray matter volumes (Haug and Eggers, 1991; Raz et al., 2004). Functional neuroimaging studies, using positron emission tomography (PET) and functional magnetic resonance (fMRI), have revealed age-related increases and decreases in task-related brain activity. These changes were initially thought to reflect poorer brain function underlying cognitive decline; decreased activation was thought to reflect a reduced allocation of neural resources, whereas increased activation was considered a marker of reduced efficiency and selectivity of neural responses, referred to as dedifferentiation (Li and Lindenberger, 1999; Li and Sikström, 2002; Grady, 2008). While dedifferentiation is still a viable explanation for some age-related neural changes, some patterns of alterations have been positively associated with cognitive function (Berlingeri et al., 2010) and have contributed to a general theory of neural compensation (Grady, 2012).

The theory of neurocompensation was initially invoked to explain observations of increased neural activity while OAs performed cognitive tasks as well as younger adults (YAs; Cabeza, 2002), or when increased activity positively correlated with performance only in OAs (McIntosh et al., 1999; ReuterLorenz et al., 2000). This view suggests that activation of alternate brain regions may serve to counteract cognitive decline (Cabeza, 2001, 2002; Cabeza et al., 2004). Evidence of agerelated neurocompensation has been found using tests of episodic retrieval (Davis et al., 2008; McDonough et al., 2012), visuospatial skills (Lee et al., 2011), WM (Rypma et al., 2007; Carp et al., 2010), response inhibition (Hsieh and Fang, 2012; Sebastian et al., 2013), and selective attention skills (Davis et al., 2011; Ansado et al., 2012). These effects are frequently reported in the prefrontal cortex (PFC; Goh, 2011), which is consistent with prior literature of neurocompensatory processes in OAs (Cabeza et al., 1997; Madden et al., 1999a,b). These findings collectively support the age-related compensatory hypothesis, although several questions remain unanswered. For example, it is unclear which cognitive processes are supported by neurocompensatory mechanisms (Rajah and D'Esposito, 2005; Greenwood, 2007; Zarahn et al., 2007).

One prominent model of age-related neurocompensation is the hemispheric asymmetry reduction in OAs (HAROLD), which postulates that under similar circumstances, PFC activation during cognitive processes tends to be less lateralized in OAs than YAs (Cabeza, 2002). HAROLD and other agerelated neurocompensatory theories offer different predictions of age-related neurocompensatory processes. For example, the compensation-related utilization of neural circuits hypothesis (CRUNCH) theory posits that the magnitude of network activation increases with task demand (Reuter-Lorenz and Cappell, 2008), while the scaffolding theory of aging and cognition (STAC) suggests a recruitment of additional networks when the primary network becomes inefficient (Park and ReuterLorenz, 2009). The HAROLD model is particularly attractive for studying age-related neurocompensation due to its focus on the PFC, which is consistent with the majority of agerelated neurocompensatory findings (see Goh, 2011 for review). Moreover, patterns of activation consistent with HAROLD have been well-validated using a range of neuroimaging paradigms, from simple motor tasks (Mattay et al., 2002) to more complex tasks, such as verbal working memory (VWM) and episodic retrieval (Bäckman et al., 1997; Cabeza et al., 1997; ReuterLorenz et al., 2000; Morcom and Friston, 2012). HAROLD thus offers a useful framework for investigating age-related patterns of neurocompensation.

While the HAROLD model has been well-documented, several aspects of this model are not fully delineated. For example, some evidence suggests that HAROLD patterns observed in prefrontal regions may be specific to higher levels of task demand (Berlingeri et al., 2013). In addition, studies assessing the HAROLD activation pattern have not consistently evaluated or found support for the relation between neurocompensatory activity and behavioral performance. For example, using PET to investigate age-related neural changes in verbal and spatial WM, OAs showed an increased bilateral response in the PFC that was associated with slower reaction time but equivalent WM accuracy. There has also been some evidence to suggest the presence of HAROLD relative to functional connectivity (FC), though with limited evidence of cognitive change (Li et al., 2009).

Another aspect of the HAROLD model that has not been clarified is the distinction between relative activation and deactivation. Despite substantial evidence for HAROLD effects in task-related activation patterns, neurocompensation relative to task-independent neural processes have been understudied. The default mode network (DMN) has been defined by coactivation within a distributed network of cortical regions, which characterizes the resting state of the human brain (Greicius et al., 2004). Evidence suggests that several properties of the DMN are sensitive to the aging process (Spreng et al., 2016; Klaassens et al., 2017). First, reduced DMN coherence has been associated with declines in processing speed ( $\mathrm{Ng}$ et al., 2016), WM (Hampson et al., 2006), and cognitive control (Turner and Spreng, 2015). Second, suppression of DMN regions (relative deactivation), which occurs during task engagement (Anticevic et al., 2012; Binder, 2012) is a marker of efficient executive functioning (EF) that becomes compromised in OAs (Duverne et al., 2008; Petrella et al., 2011; Spreng et al., 2016; Avelar-Pereira et al., 2017).

Evidence suggests that DMN deactivations may be particularly susceptible to aging and a potential contributor to neurocompensatory processes. For example, using fMRI and spatial judgment tasks, Park et al. (2010) reported significantly reduced deactivations in OAs, and importantly, faster reaction times were demonstrated by OAs who did effectively deactivate DMN regions. In addition, Miller et al. (2008) reported enhanced DMN deactivation in YAs that correlated with successful memory encoding; however, OAs did not show this pattern, and reduced deactivation was most evident among poorer performers. Using a verb generation task, Persson et al. (2007) also reported an age-related reduction in DMN deactivations that was associated with slower reaction time performance. Perhaps the strongest evidence of DMN-related neurocompensation is described by 
Davis et al. (2008), who developed the posterior-anterior shift in aging (PASA) hypothesis to explain the observation that OAs showed a pattern of reduced deactivations of posterior midline cortex, with increased deactivations of medial frontal cortex during a verb generation task. When matching groups on performance, OAs showed greater DMN deactivation of the left anterior cingulate and right anterior insula, as well as increased activations in the left middle frontal gyrus and right supramarginal gyrus. Both deactivation and activation effects were seen bilaterally, which, in the context of the relation between the DMN and task-positive network functions, suggests that the deactivations may also support cross-hemispheric neurocompensatory processes. Importantly, given the inverse correlation between DMN and task-positive networks that supports cognitive functioning (Fox et al., 2005), these bilateral effects suggest that an age-related neurocompensatory process (i.e., HAROLD) of relative deactivations may support successful task-positive functioning.

Further investigations of task-independent processes are needed to determine how the DMN and task-independent networks may interact with other models of neurocompensation, such as the HAROLD pattern in OAs. Since HAROLD and PASA models do not have direct support from studies of relative deactivation and given a lack of clarity in prior literature whether effects included summation of activation and relative deactivation or only activation effects, we considered examination of activation and relative deactivation separately to have high theoretical and methodological importance. The present study provides the first examination of HAROLD lateralization effects in both brain activations and relative deactivations in healthy OAs. Using fMRI during a WM paradigm, we evaluated the presence of the HAROLD pattern in the task-dependent brain response because neural activation associated with VWM has previously demonstrated sensitivity to age-related neurocompensatory processes, including the HAROLD effects. Second, using a resting fixation baseline to isolate suppression of baseline DMN activity, we evaluated the presence of HAROLD among task-induced deactivations. Moderation analyses were conducted to address whether observed HAROLD effects were associated with cognitive performance (i.e., n-back accuracy). Two homologous frontal lobe regions of interest (ROI) were used to calculate the laterality indices (LI) and examine the expected HAROLD effects. It was hypothesized that (1) OAs would demonstrate HAROLD patterns of task-elicited relative brain activation and deactivations, and (2) effects would be significantly moderated by 2-back performance, as a reflection of successful compensation.

\section{MATERIALS AND METHODS}

\section{Participants}

Participants included 45 healthy, right handed English-speaking men and women over the age of 50 (25 women, age range $53-83, M$ age $=63.78$ years, $S D=7.99$ ) who comprised a healthy control group in a larger study of neurocognitive function in cardiovascular disease (Haley et al., 2009). These participants were recruited from the community via advertisements in the Providence, RI area. All participants underwent standard informed and written consent procedures. Assessments were conducted over three visits that spanned approximately 6 weeks and included a neuropsychological assessment, an echocardiogram, and an MRI scanning session. Exclusion criteria included left-hand dominance, corrected visual acuity poorer than 20:40, below $60 \%$ performance accuracy on the 2-back VWM task (i.e., < 1 SD from 50\%), low global cognitive function ( $>1.5 \mathrm{SDs}$ below the sample population on the Mini Mental Status Examination), or any MRI contraindications (e.g., metal implants). Significant medical (e.g., surgery, heart infarct), neurological (e.g., multiple sclerosis, traumatic brain injury with loss of consciousness), and psychiatric problems (e.g., substance abuse with hospitalization, diagnosis of any current psychiatric illness) were exclusion criteria that were assessed by interview, physical examination, review of medical records and self-report questionnaires. Participants were compensated for their participation. The study was approved and monitored by the university and hospital institutional review boards (IRB) where the research took place and conformed to the Helsinki Declaration on human subjects' protection.

Demographic characteristics, estimated intellectual functioning, and 2-back performance are displayed in Table 1. The study sample comprised OAs with right-handed dominance $(M=86.7, S D=14.40$; range $=55-100 ;$ on a scale of $0-100$, with increasing values representative of increasing right-dominance) and above average intellectual functioning (WTAR range $=96-$ 119 , mean $=111.53, S D=6.40$ ) and years of educational attainment (range $=12-21$ years; mean $=16.36 ; S D=1.96)$. WM performance (i.e., 2-back accuracy) was consistent with prior 2-back literature (Braver et al., 1997; Smith and Jonides, 1999; Sweet et al., 2008).

\section{Behavioral Measures}

Verbal working memory paradigm. The n-back paradigm was employed to challenge VWM systems during two imaging runs. The n-back has been widely used in functional neuroimaging research for more than 20 years and has the advantage of a welldescribed fMRI neural response (e.g., Braver et al., 1997; Smith and Jonides, 1999; Owen et al., 2005; Sweet et al., 2008). During

TABLE 1 | Demographic and mean summary data.

\begin{tabular}{lccrr}
\hline Variable & Mean & SD & Min & Max \\
\hline Age (years) & 63.78 & 7.99 & 53.00 & 83.00 \\
MMSE & 29.40 & 0.70 & 28.00 & 30.00 \\
Handedness (\%R) & 86.7 & 14.40 & 55.00 & 100.00 \\
Education (years) & 16.36 & 1.96 & 12.00 & 21.00 \\
Predicted FSIQ & 111.5 & 6.40 & 96.00 & 119.00 \\
2-back accuracy & 82.98 & 9.70 & 64.00 & 99.00 \\
\hline
\end{tabular}

MMSE, Mini mental status exam; Handedness was measured on a scale of 1-100, with increasing values representative of right-handed dominance, and all subjects reported right-handedness; Education, years of formal education attained; FSIQ, Full-scale intelligence quotient from the Wechsler Test of Adult Reading; 2-back accuracy includes correct hits and rejections proportional to the number of trials. 
the 2-back, six series of 15 consonants were presented visually for $500 \mathrm{~ms}$ each, with an interstimulus interval (ISI) of $2500 \mathrm{~ms}$. Participants were asked to make a yes or no button-press response following each consonant to report whether or not it was the same as the consonant (irrespective of capitalization) presented two earlier in the series (e.g., underlined letters in the following sequence would be answered "yes": w, N, r, $\underline{N}, \underline{R}, Q, \underline{r}, \underline{q}, N, W$, $\underline{\mathrm{n}}$...). Six 0-Back control blocks of nine consonants each were presented with the same duration of letter presentation and ISI. 0Back blocks preceded each 2-Back block during the first imaging run and followed the 2-Back during the second run. Participants responded yes when a predetermined target consonant (" $\mathrm{H}$ " or " $h$ ") appeared and no for other consonants. Consonant blocks of both conditions contained 33 percent "yes" targets in random locations within each series. Capitalization was randomized throughout to encourage verbal encoding. Two 27-s (27000 ms) blocks of resting fixation blocks were presented between the 0-Back/2-Back cycles. 2-back performance was calculated for each participant using the following formula: (number of correct positives + correct negatives)/ total number of letters presented. Two subjects were excluded from behavioral analyses due to nearchance 2 -back performance ( $<60 \%$ accuracy). A diagram of the $\mathrm{n}$-back task is presented in Supplementary Materials.

\section{Neuroimaging Measures}

MRI Acquisition. Whole-brain echo-planar fMRI was conducted using a Siemens TIM Trio 3 tesla scanner $(T R=2500 \mathrm{~ms}$, $T E=28 \mathrm{~ms}, F O V=192^{2}$, matrix size $=64^{2}$, in 423 -mm-thick axial slices). This procedure yielded 116 whole-brain volumes for each of the two $288 \mathrm{~s}$ imaging runs, yielding a spatial resolution of $3 \mathrm{~mm}^{3}$ per voxel. Whole-brain high-resolution T1 images were also acquired in the sagittal plane for anatomical reference $\left(T R=1900 \mathrm{~ms}, T E=2.98 \mathrm{~ms}, F O V=256^{2} \mathrm{~mm}\right.$, matrix size $256^{2}$ ). Two conditions of the n-back paradigm (i.e., 2-back and 0 -back) and a resting state "+" were presented using E-prime (Psychology Software Tools, Sharpsburg, MD, United States) and back-projected onto a screen visible to the participant via a mirror mounted to the head coil.

MRI analysis. MRI dataset processing and statistical analyses were performed with Analysis of Functional NeuroImages version 18.0.05 software (AFNI; Cox, 1996). Preprocessing of the functional runs included slice-time correction and registration of each volume to the third volume of the first imaging run to correct for head movement. Data from participants with head movement of $>3.0 \mathrm{~mm}$ in any direction (i.e., $\mathrm{x}, \mathrm{y}, \mathrm{z}, \mathrm{yaw}$, pitch, and roll) or movement greater than $0.3 \mathrm{~mm}$ on more than $25 \%$ of repetitions were omitted from analyses. The functional volumes were aligned to the anatomical volume in Talairach space. A 5$\mathrm{mm}$ full-width half maximum Gaussian filter was applied and the raw time-series was scaled to a mean of 100 . For each subject, the general linear model (GLM) was used to quantify 2back activity relative to a resting state (i.e., fixation across) after controlling for 0 -back active control task and other covariates (i.e., movement parameters) in order to facilitate examination of task-independent deactivations.

Individual activity maps. The resulting individual activity maps were thresholded (two-tailed $\alpha=0.01$ ) and corrected for multiple comparisons using AFNI's false discovery rate (FDR; $q=0.05$ ) procedure in order to provide a measure of volumetric activity, measured by significantly activated voxels beyond the threshold per ROI, for each participant.

Group level processing. For group level-analyses, two frontal ROIs were defined based on left or right hemisphere (i.e., relative to Talairach coordinate $x$-plane $=0$ ) which extended to $y$-plane $=0$. LIs, defined as left relative to right (left/right) frontal ROI response to the 2-back were calculated using each individual's volume of significant response in each ROI (i.e., voxels of significant activity beyond threshold). Consistent with prior literature (Deblaere et al., 2004; Baciu et al., 2005; Yuan et al., 2006), an LI was calculated by subtracting intensity effects within the right frontal ROI from intensity effects within the left frontal ROI, then dividing this value by the average of mean activity across the two frontal ROIs (i.e., (left frontal right frontal) / (left + right frontal). Thus, the scaling of LI was such that positive values indicate left-lateralized function and negative values indicate right-lateralized function. This process was conducted separately for relative activation and deactivation and repeated for volumes of significant activation and deactivation.

Group level analyses. Qualitative procedures were first conducted to examine the validity of brain activation patterns exhibited by the sample. In order to generate relative group level activation and deactivation maps for comparison to prior literature, the whole-brain unthresholded voxel-wise effects were tested against a hypothetical mean of zero (i.e., no 2-back effect) using one-sample $t$-tests. These whole-brain analyses were conducted using an FDR-corrected threshold of $p=10^{-9}$ (voxels greater than $t=8.42$ ) with and a minimum of 10 contiguous voxels. Clusters of significant n-back response exhibited by our sample was compared to prior n-back literature (e.g., Smith and Jonides, 1999; Owen et al., 2005); in addition, the Neurosynth Image Decoder was used to quantify the concordance between the whole-brain results and neuroimaging studies included in the publicly available database. Next, hemispheric lateralization was examined. Consistent with prior literature, hemispheric dominance was determined by the size of the LI (Deblaere et al., 2004; Baciu et al., 2005; Yuan et al., 2006), following the criteria adopted by Yuan et al., 2006, in which an LI threshold of 0.10 (representative of 10 percent greater left relative to right hemispheric activity) was considered evidence of lateralization. Lastly, support for HAROLD effects were assessed by performing bivariate correlation analyses between age and each LI.

Behavioral analyses. Hierarchical multiple regression and moderation analyses were conducted using the Statistical Package for Social Sciences IBM SPSS Statistics 21.0 to test the influence of 2-back accuracy on HAROLD effects. Before conducting moderation analyses, assumptions of multiple linear regressions were examined, including homoscedasticity, independence of residuals and normality of residuals (Cohen et al., 2003). Multicollinearity between the independent variable and moderator were reduced through the use of centering (Aiken and West, 1991). For moderation analyses, the PROCESS SPSS macro plug-in (Hayes, 2012) was applied to examine the moderating influence of $\mathrm{n}$-back performance on the relation 


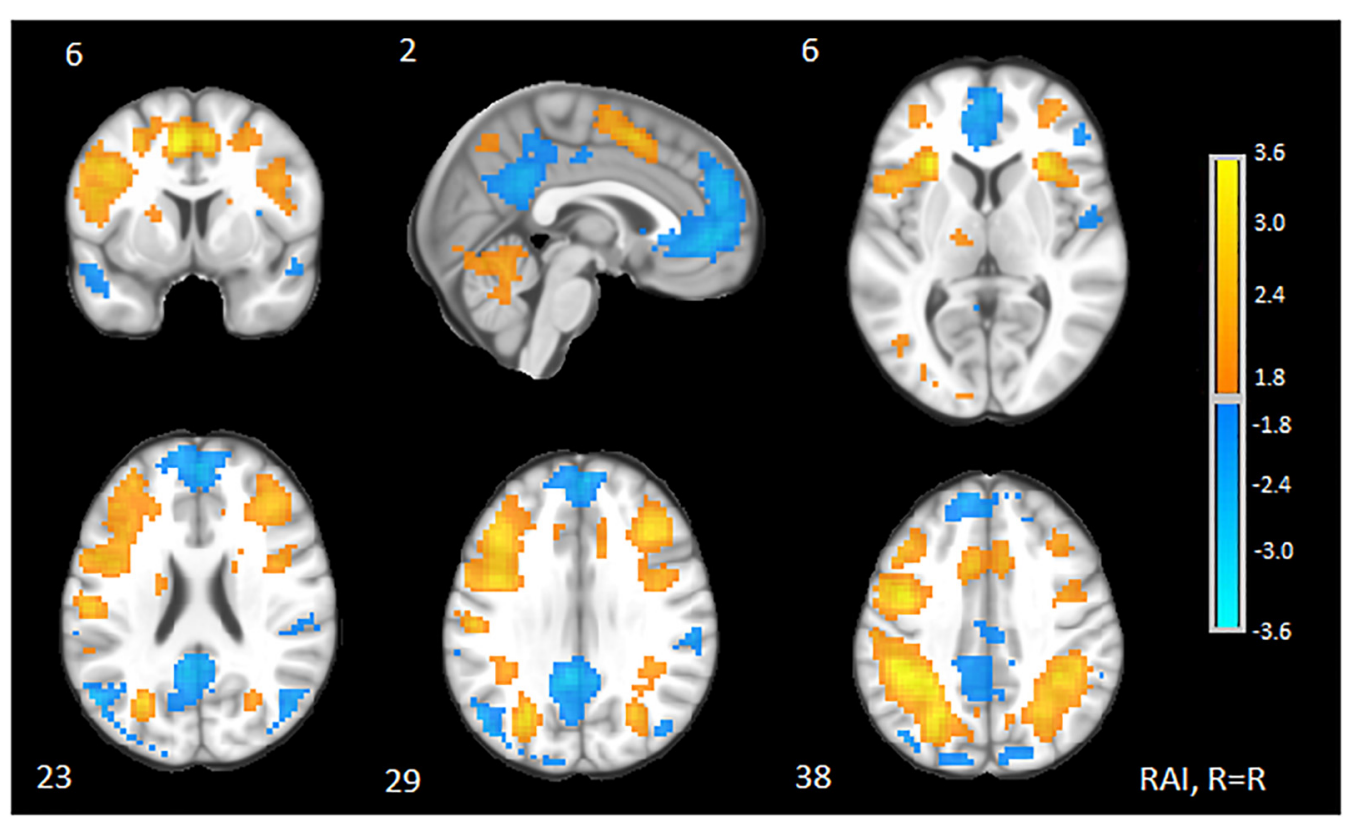

FIGURE 1 | Clusters of significant neural response to 2-back. Representative of 2-back task-based activations and deactivations relative to a resting state baseline (“+”) from GLM analysis. Slices are provided in Talairach space and shown in RAl orientation (right = right). Yellow, activations; Blue, deactivations. Cluster centers of mass are reported in Table 2. Slice number $(Z)$ is located above each slice. Color bars indicate magnitude effects for activations and deactivations.

between age and LI. For the present study, the interactions were visually probed in order to examine the nature of any moderation effects by examining conditional effects (i.e. simple slopes) at low $(-1 S D$ below the mean) and high $(+1 S D$ above the mean) levels of 2-back performance.

\section{RESULTS}

\section{Whole Brain Voxelwise Analyses}

The 2-back versus baseline contrast revealed widespread activation patterns commonly associated with n-back performance (Smith and Jonides, 1999; Owen et al., 2005), including the bilateral middle frontal gyrus, medial frontal gyrus, inferior parietal cortices, insula, and cerebellum. Relative deactivations were also consistent with prior literature (Anticevic et al., 2012; Binder, 2012) and overlapped substantially with regions associated with the DMN (Buckner et al., 2008), including the medial frontal gyrus/anterior cingulate, posterior cingulate, and superior frontal gyrus. Results from the Neurosynth Decoder, which was used to quantify the concordance of the present results to those in the publicly available database, indicated a value of 0.32 that was most consistent with taskspecific measures of WM. See Figure 1 and Table 2 for neural patterns and related activity maps.

\section{Laterality and HAROLD Effects}

Volumetric effects of the 2-back were summarized by the two frontal ROIs for both activations and deactivations. LIs were then calculated from these values and averaged at the group level. The activation LI yielded left-lateralization of this VWM task
TABLE 2 | Clusters of significant neural response to 2-back versus resting state.

\begin{tabular}{lcccc}
\hline Region & Voxels & $\mathbf{x}$ & $\mathbf{y}$ & $\mathbf{z}$ \\
\hline L inferior parietal lobule & 239 & 34 & 50 & 41 \\
B posterior cingulate & 231 & 0 & -47 & 10 \\
R cerebellum & 151 & -26 & 53 & -22 \\
L medial frontal gyrus & 57 & 5 & -5 & 51 \\
L precentral/postcentral gyrus & 51 & 35 & 22 & 53 \\
B medial frontal & 46 & 2 & 48 & 30 \\
L inferior frontal/precentral gyrus & 45 & 42 & -3 & 33 \\
L insula & 37 & 29 & -22 & 9 \\
R inferior parietal lobule & 36 & -42 & 44 & 41 \\
R insula & 34 & -31 & -23 & 6 \\
R medial frontal gyrus & 25 & -9 & -15 & 45 \\
L cerebellum & 21 & 32 & 50 & -26 \\
R middle frontal gyrus & 21 & -39 & -29 & 29 \\
R superior parietal lobule & 20 & -30 & 60 & 42 \\
L middle frontal gyrus & 14 & 25 & 6 & 50 \\
\hline
\end{tabular}

L, Left; R, Right; B, Bilateral. Coordinates reported in center of mass Talairach space, RAl orientation. These clusters are shown in Figure 1.

$(M=0.15, S D=0.19)$ as expected (Jonides et al., 1997). Similarly, the relative deactivation $L I$ was left-lateralized $(M=0.17$, $S D=0.19$ ). Descriptive statistics and the computed LI values are presented in Table 3.

The presence of HAROLD was then examined via bivariate Pearson correlations between age and each LI. The LI of neural activations was significantly and positively associated with age for volumetric effects $r(42)=0.34, p=0.02$ (see Table 4). Consistent with the HAROLD model predictions, the LI of neural 
TABLE 3 | Neural effects of relative activations, deactivations, and laterality indices (LIs).

\begin{tabular}{lccc}
\hline Regions & Mean & SD & LI \\
\hline Activations & 606 & 395 & $0.12^{*}$ \\
Left Frontal & 478 & 324 & \\
Right Frontal & & & $0.17^{*}$ \\
Deactivations & 511 & 310 & \\
Left Frontal & 363 & 233 & \\
Right Frontal & & & \\
\hline
\end{tabular}

ROls, Regions of interest relative to task-induced activations and deactivations. Mean, Mean number of significantly active voxels within each frontal ROl; SD, Standard deviation of significantly active voxels within each frontal ROI. *Denotes left-lateralization of LI (Yuan et al., 2006).

TABLE 4 | Correlations among demographics, cognition, and LI.

\begin{tabular}{lccc}
\hline Variable & Age & Education & n-back \\
\hline Activations & & \\
Age & & \\
Education & -0.02 & 0.05 & \\
n-back & -0.18 & 0.08 & -0.15 \\
Laterality Index & $0.34 *$ & \\
Deactivations & & \\
Age & & \\
Education & -0.02 & 0.04 & $0.37^{*}$ \\
n-back & -0.18 & 0.14 & \\
Laterality Index & $-0.31 *$ & \\
\hline * $p$ 0.05. A significant relation between age and the laterality index of neural \\
deactivations was interpreted as consistent with the HAROLD effects.
\end{tabular}

deactivations were significantly and negatively associated with age $r(42)=-0.31, p=0.04$ (see Table 4). Scatter plots of the relation between age and LIs for relative activations and deactivations are presented in Figures 2, 3, respectively, with residuals shown in Supplementary Materials.

\section{Moderating Effect of 2-Back Accuracy}

Hierarchical multiple regression and moderation analyses were conducted to examine the influence of 2-back performance on the relation between age and LIs. Two models were used to explore the four statistically significant relations between age and LIs of relative activations and deactivations.

For neural activations, age significantly predicted LI $(b=0.01$, $p=0.03)$. However, 2-back accuracy $(b=0.00, p=0.98)$, and the age $\times 2$-back accuracy interaction term $(b=0.00, p=0.96)$ were not significantly predictive of LI (total $R^{2}=0.13, p=0.96$ ). Moderation analysis specific to neural deactivations, however, yielded significant effects. Over and above age $(b=-0.01$; $p=0.02), 2$-back accuracy $(b=0.01 ; p=0.05)$ was a significant predictor of unique variance in LI (total $R^{2}=0.28 ; F(3,40)=5.09$, $p<0.001)$. In addition, the interaction term of 2-back accuracy $\mathrm{x}$ age was a significant predictor of unique variance in LI over and above age and 2-back accuracy $\left(\Delta R^{2}=0.08 ; \Delta F(3,40)=4.33\right.$; $b=0.00 ; p=0.04)$. Results of this moderation effect are presented in Table 5.
Aging and Increased Left-lateralization of Neural Activations

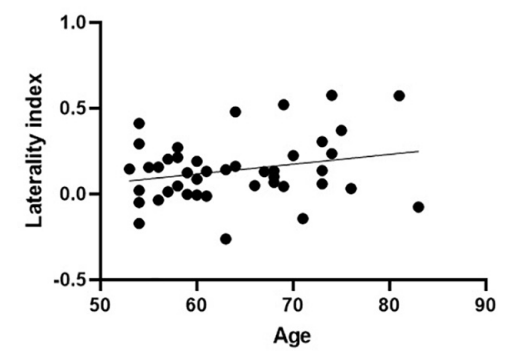

FIGURE 2 | Aging and increased left-lateralization of neural activations. A significant and positive relationship between age and laterality index of neural activations was not considered consistent with the neural pattern of the hemispheric asymmetry reduction in older adults (HAROLD) effect.

Aging and Reduced Left-lateralization of Neural Deactivations

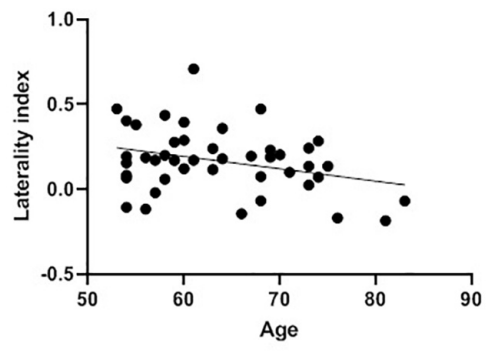

FIGURE 3 | Aging and reduced left-lateralization of neural deactivations. A significant and positive relationship between age and laterality index of neural deactivations was considered consistent with the neural pattern of the hemispheric asymmetry reduction in older adults (HAROLD) effect.

Given evidence of an interaction effect between age and 2-back accuracy on the relative deactivation LI, simple slopes analyses were conducted by estimating the conditional effect of age at specific values of 2-back performance (in this case, \pm 1 SD from the sample mean, or -11.50 and 11.50 , respectively) and tested whether the slopes were statistically significant from zero by a null hypothesis test (see Hayes, 2013). Results of these analyses revealed an association between age and LI, with age significantly related to LI for higher levels of 2-back performance $(b=-0.01, p=0.01,95 \% C I s=-0.05,-0.00)$ but not significantly related to LI for lower levels $(b=-0.00$, $p=0.82,95 \% C I s=-0.01,0.01)$. In other words, high 2-back performers exhibited a stronger negative relationship between age and deactivation LI, or HAROLD effects. A plot of the simple slopes analyses is presented in Figure 4.

\section{DISCUSSION}

Hemispheric asymmetry reduction in older adults is a well-established neurocompensatory process that has been consistently demonstrated to support healthy OAs maintenance of cognitive function (Reuter-Lorenz et al., 2000; Cabeza, 2002; Morcom and Friston, 2012). However, to date, no studies have 
TABLE 5 | 2-back accuracy moderates age and deactivation laterality.

\begin{tabular}{|c|c|c|c|c|c|c|}
\hline Total Model & $\mathbf{R}^{2}$ & $S E$ & $\mathbf{F}$ & $p$ & $\Delta \mathbf{R}^{2}$ & \\
\hline & 0.28 & 0.03 & 5.09 & 0.00 & 0.08 & \\
\hline Model & $b$ & SE & $t$ & $p$ & LLCl & ULCI \\
\hline \multicolumn{7}{|l|}{ Main Effects } \\
\hline Age & -0.01 & 0.00 & -2.33 & 0.02 & -0.01 & 0.00 \\
\hline n-back & 0.01 & 0.00 & 2.02 & 0.05 & 0.00 & 0.01 \\
\hline \multicolumn{7}{|l|}{ Interaction } \\
\hline Age * n-back & 0.00 & 0.00 & -2.08 & 0.04 & 0.00 & 0.00 \\
\hline
\end{tabular}

$* p<0.05$.

investigated HAROLD effects on relative deactivations of the DMN. The present study sought to determine if (a) HAROLD would generalize to task-induced activations and deactivations using a verbal n-back paradigm, and (b) if HAROLD effects would be significantly and negatively associated with 2-back performance. Results of whole-brain voxelwise analyses revealed expected patterns of neural response associated with the 2back vs. a resting state baseline (Owen et al., 2005), including deactivations in DMN regions (Buckner et al., 2008). LIs calculated from 2-back-associated neural activation indicated left-lateralization that was consistent with previous findings (Smith and Jonides, 1999; Owen et al., 2005). Extending this finding, the deactivation LI also indicated left-lateralization. Regarding neurocompensatory processes, OAs demonstrated an unexpected age-related increase in left-lateralized activity that was not overtly associated with 2-back performance. In contrast, and consistent with our second hypothesis, an age-related change in the deactivation LI reflected a HAROLD pattern that was influenced by 2-back performance, such that high- but not low-performers demonstrated this shift.

The significant positive correlation between age and activation LI suggests that an age-related increase in left hemispheric brain response may have supported OAs' maintenance of cognitive functioning. While this finding appears to contradict the HAROLD model, it is consistent with evidence supporting the presence of other neurocognitive compensatory processes in OAs. For example, differences in capacity (i.e., the degree to which a brain network is maximally recruited to perform a task) has been associated with cognitive maintenance (Bosch et al., 2010). At the network level, the compensation-related utilization of neural circuits hypothesis (CRUNCH) theory predicts that increased cognitive demands will result in a concomitant increase in network function (Reuter-Lorenz and Cappell, 2008). In the same vein, evidence has supported the STAC, which posits that recruitment of additional neural circuits or even networks occur when the primary networks have become inefficient or damaged due to pathology (Park and Reuter-Lorenz, 2009). Thus, one or more broader theories of compensation may complement the HAROLD model as it relates to successful maintenance of VWM. The younger age range of the present sample, relative to the age of participants in prior HAROLD studies (Cabeza, 2002) is not inconsistent with more traditional compensatory models.

Uncertainty regarding the function of overactivation, however, has led to increased interest in cognitive performance

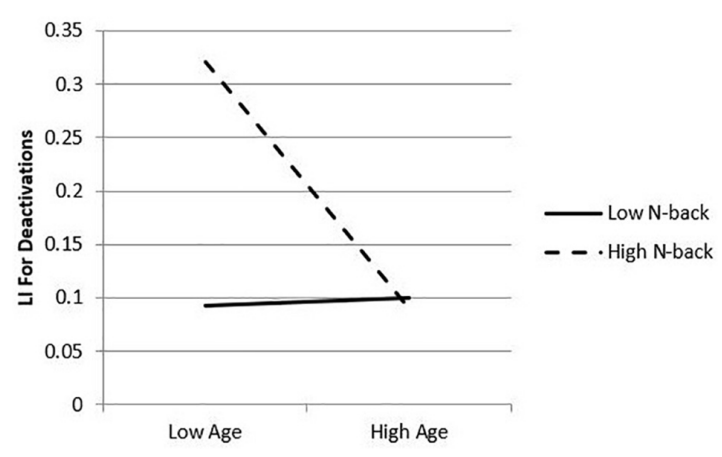

FIGURE 4 | Simple slopes plot of conditional effects of 2-back. 2-back performance moderates the relation between age and laterality index. Plot represents laterality index (greater number depicts greater left lateralization) as predicted by age at fixed values of the moderator, 2-back: +1 standard deviation (11.5) and -1 standard deviation $(-11.5)$ from the sample mean.

correlates. For example, overactivation among OAs has been linked to both reduced (Logan et al., 2002; Milham et al., 2002; Johnson et al., 2004; Park et al., 2004; Thomsen et al., 2004) and maintained cognitive performance (Grady et al., 2003; Persson et al., 2004; Rossi et al., 2004; Gutchess et al., 2005) which have been interpreted to reflect dedifferentiation and compensation, respectively. In the present study, 2-back accuracy did not moderate the relation between age and LI for activations, suggesting that increased left-lateralized activity did not function to maintain performance. However, lack of an observable relation between overactivation and performance may not necessarily mean that these alterations are not compensatory in nature; for example, compensatory increases in bilateral recruitment may not be extensive enough to offset opposite-hemisphere reduction in efficiency (i.e., resulting in performance declines), or individual differences may not be related to performance on a specific task (see Grady, 2012 for review). Importantly, while higher performing OAs in the current study did not evidence a greater degree of overactivation, lower performing OAs also did not. It is therefore plausible that the lower performing OAs maintained previous levels of cognitive function prior to age-related decline via increased left-lateralized activation.

Findings of the present study support the notion that relative deactivations are sensitive to the aging process, lateralized similarly to task-induced activations, and associated with 
successful task performance on a measure of VMW. A significant negative correlation between age and the deactivation LI, coupled with maintained 2-back performance (i.e., > $>60 \%$ for each participant) suggests that reduced asymmetry of deactivations supported the OAs' maintenance of cognitive functioning. Therefore, the current results provide evidence of a novel HAROLD finding, extending previous findings to deactivations, and more specifically, via a within-group fMRI study of community-dwelling OAs. This finding is further strengthened by an observed moderation effect of 2-back accuracy, suggesting that reduced asymmetry of deactivations may support maintenance of baseline cognitive abilities. Results of simple slopes analyses, conducted to elucidate the nature of this effect, revealed that higher performing OAs demonstrated an increased reduction of asymmetry specific to deactivations, while lower performing OAs did not (see Figure 2). This is consistent with the growing expectation that neurocompensatory processes relate to an observable maintenance in cognition.

Due in part to observations of their importance in neurocognitive function, task-induced deactivations of the DMN have become the target of increased investigation (Anticevic et al., 2012; Binder, 2012; Gilbert et al., 2012; Spreng, 2012; Hansen et al., 2014; Spreng et al., 2016). Evidence suggests that deactivations are reduced in the aging process (Lustig et al., 2003; Rombouts et al., 2005; Grady et al., 2006) and associated with poorer task performance (Persson et al., 2007). The present findings suggest that DMN deactivations may also help to explain neurocompensatory processes. This notion is further substantiated by the relation between DMN and taskpositive network functioning (Anticevic et al., 2012; Spreng et al., 2016); for example, cross-hemispheric neurocompensatory activations (e.g., HAROLD) may correspond with crosshemispheric alterations in DMN deactivation patterns that are required for effective reallocation of neural resources.

Consistent with this notion, the PASA model has been used to explain an age-related posterior-anterior shift of task-related DMN deactivations that appear to support cognitive function, such as semantic fluency (Davis et al., 2008). Others have since identified general age-related reductions in DMN deactivation that were associated with slower reaction time on measures of spatial skills and higher scores on tests of EF (Persson et al., 2007; Park et al., 2010). Results of the present study provide further evidence that DMN deactivations may be particularly important for the maintenance of cognitive function in the face of age-related decline and warrant future investigations of neurocompensatory processes.

\section{Limitations}

Few studies have investigated HAROLD effects specific to WM among healthy OAs, and most were older PET studies. Prior literature has also focused almost exclusively on group differences (i.e., YA vs. OA) in HAROLD effects specific to task-dependent activations associated with WM. Interpretation of our findings by comparison to prior studies is therefore limited. A YA comparison group would have aided the interpretation of results. In addition, the sample of the present study was relatively young with respect to the aging literature (range $=53$ to 83 years; mean $=63.78, S D=7.99)$, as researchers often sample OA populations from 65 years and beyond. Interpretation of study findings are also limited by a relatively small sample size (i.e., not adequately powered to detect small effects). The sample was above average in intelligence, well-educated, and predominantly of Caucasian ethnicity (96\%), which poses potential problems with generalizability.

\section{Future Directions}

As the first investigation of age-related task-independent neurocompensatory processes utilizing a within-group experimental design, findings of the current study warrant replication. Consistent with the $\mathrm{CRUNCH}$ model and a prior study of HAROLD (Berlingeri et al., 2013), results of the present study suggest that multiple compensatory processes may support cognitive function in old age. Given our identification of dual neurocompensatory processes (i.e., bilateralization of task-independent deactivations and increasing left-lateralization of task-related activations) during a VWM paradigm, future investigations may also benefit from consideration of both taskdependent and task-independent brain responses in the context of newer models of neurocompensation such as CRUNCH and STAC. Evidence of HAROLD effects among relative to deactivations suggest that baseline DMN processing may be altered in OAs. Therefore, decline in cognitive performance and associated task-related brain response may be explained, in part, by changes in baseline processing.

A better understanding of factors that influence neurocompensatory processes may aide healthy OAs in maintaining cognitive function and aging gracefully. For example, several sociological and cultural factors have been linked to maintenance of cognitive function in aging, such as education, occupational complexity, social activity, and physical exercise (Katzman, 1993; Valenzuela et al., 2008; Stern, 2012), and brain changes associated with these factors is a topic of growing interest (Barulli and Stern, 2013; Stern, 2016). Future research of age-related neurocompensatory processes may also aide researchers and clinicians in identifying problem areas in OAs (e.g., less efficient processing, overactivations and performance declines), and those at-risk of developing neurodegenerative conditions. Early identification of at-risk OAs may in turn inform developing cognitive training programs, which refer to a range of structured programs (e.g., computerized tasks engaging EF systems) intended to maintain cognition or ameliorate cognitive deficits (Bahar-Fuchs et al., 2013). A growing literature suggests that cognitive (Karbach and Verhaeghen, 2014) and physical exercise interventions (Bherer, 2015) induce neural changes associated with improved cognitive performance (for review, see Bamidis et al., 2014) and appear to be a promising future complement to pharmacological interventions for OAs and warrants continued research.

\section{ETHICS STATEMENT}

The study was approved and monitored by the university and hospital institutional review boards (IRB) where the research 
took place and conformed to the Helsinki Declaration on human subjects' protection. All participants underwent standard informed and written consent procedures.

\section{AUTHOR CONTRIBUTIONS}

$\mathrm{BD}, \mathrm{MO}, \mathrm{EH}$, and LS contributed to the design and implementation of the research, analysis of the results, and writing of the manuscript.

\section{REFERENCES}

Aiken, L., and West, S. (1991). Testing and Interpreting Interactions. Newbury Park, CA: Sage Publications.

Alzheimer's Disease International (2010). World Alzheimer Report. Available at: http://www.alz.co.uk/research/world-report (accessed April 19, 2018).

Ansado, J., Monchi, O., Ennabil, N., Faure, S., and Joanette, Y. (2012). Loaddependent posterior-anterior shift in aging in complex visual selective attention situations. Brain Res. 1454, 14-22. doi: 10.1016/j.brainres.2012.02.061

Anticevic, A., Cole, M. W., Murray, J. D., Corlett, P. R., Wang, X. J., and Krystal, J. H. (2012). The role of default network deactivation in cognition and disease. Trends Cogn. Sci. 16, 584-592. doi: 10.1016/j.tics.2012.10.008

Avelar-Pereira, B., Bäckman, L., Wåhlin, A., Nyberg, L., and Salami, A. (2017). Agerelated differences in dynamic interactions among default mode, frontoparietal control, and dorsal attention networks during resting-state and interference resolution. Front. Aging Neurosci. 9:152. doi: 10.3389/fnagi.2017.00152

Baciu, M. V., Watson, J. M., Maccotta, L., McDermott, K. B., Buckner, R. L., and Gilliam, F. G. (2005). Evaluating functional MRI procedures for assessing hemispheric language dominance in neurosurgical patients. Neuroradiology 47 , 835-844. doi: 10.1007/s00234-005-1431-3

Bäckman, L., Almkvist, O., Andersson, J., Nordberg, A., Windblad, B., Rineck, R., et al. (1997). Brain activation in young and older adults during implicit and explicit retrieval. J. Cogn. Neurosci. 9, 378-391. doi: 10.1162/jocn.1997.9.3.378

Bahar-Fuchs, A., Clare, L., and Woods, B. (2013). Cognitive training and cognitive rehabilitation for mild to moderate Alzheimer's disease and vascular dementia. Cochrane Database Syst. Rev. 6:CD003260.

Balota, D. A., Dolan, P. O., and Duchek, J. M. (2000). "Memory changes in healthy older adults," in The Oxford Handbook of Memory, eds E. Tulving and F. I. M. Craik (New York, NY: Oxford University Press), 395-409.

Bamidis, P. D., Vivas, A. B., Styliadis, C., Frantzidis, C., Klados, M., Schlee, W., et al. (2014). A review of physical and cognitive interventions in aging. Neurosci. Biobehav. Rev. 44, 206-220. doi: 10.1016/j.neubiorev.2014.03.019

Barr, R. A., and Giambra, L. M. (1990). Age-related decrement in auditory selective attention. Psychol. Aging 5:597. doi: 10.1037/0882-7974.5.4.597

Barulli, D., and Stern, Y. (2013). Efficiency, capacity, compensation, maintenance, plasticity: emerging concepts in cognitive reserve. Trends Cogn. Sci. 17, $502-$ 509. doi: 10.1016/j.tics.2013.08.012

Berlingeri, M., Bottini, G., Danelli, L., Ferri, F., Traficante, D., Sacheli, L., et al. (2010). With time on our side? Task-dependent compensatory processes in graceful aging. Exp. Brain Res. 205, 307-324. doi: 10.1007/s00221-010-2363-7

Berlingeri, M., Danelli, L., Bottini, G., Sberna, M., and Paulesu, E. (2013). Reassessing the HAROLD model: Is the hemispheric asymmetry reduction in older adults a special case of compensatory-related utilisation of neural circuits? Exp. Brain Res. 224, 393-410. doi: 10.1007/s00221-012-3319-x

Bherer, L. (2015). Cognitive plasticity in older adults: effects of cognitive training and physical exercise. Ann. N.Y. Acad. Sci. 1337, 1-6. doi: 10.1111/nyas.12682

Binder, J. R. (2012). Task-induced deactivation and the" resting" state. NeuroImage 62, 1086-1091. doi: 10.1016/j.neuroimage.2011.09.026

Bosch, B., Bartrés-Faz, D., Rami, L., Arenaza-Urquijo, E. M., Fernández-Espejo, D., Junqué, C., et al. (2010). Cognitive reserve modulates task-induced activations and deactivations in healthy elders, amnestic mild cognitive impairment and mild Alzheimer's disease. Cortex 46, 451-461. doi: 10.1016/j.cortex.2009.05.006

Braver, T. S., Cohen, J. D., Nystrom, L. E., Jonides, J., Smith, E. E., and Noll, D. C. (1997). A parametric study of prefrontal cortex involvement in

\section{ACKNOWLEDGMENTS}

This work was supported by R01HL084178-01.

\section{SUPPLEMENTARY MATERIAL}

The Supplementary Material for this article can be found online at: https://www.frontiersin.org/articles/10.3389/fnagi. 2019.00111/full\#supplementary-material

human working memory. Neuroimage 5, 49-62. doi: 10.1006/nimg.1996. 0247

Buckner, R. L., Andrews-Hanna, J. R., and Schacter, D. L. (2008). The brain's default network. Ann. N.Y. Acad. Sci. 1124, 1-38. doi: 10.1196/annals.1440.011

Cabeza, R. (2001). Cognitive neuroscience of aging: contributions of functional neuroimaging. Scand. J. Psychol. 42, 277-286. doi: 10.1111/1467-9450.00237

Cabeza, R. (2002). Hemispheric asymmetry reduction in older adults: the HAROLD model. Psychol. Aging 17, 85-100. doi: 10.1037/0882-7974.17.1.85

Cabeza, R., Daselaar, S. M., Dolcos, F., Prince, S. E., Budde, M., and Nyberg, L. (2004). Task-independent and task- specific age effects on brain activity during working memory, visual attention and episodic retrieval. Cereb. Cortex 14, 364-375. doi: 10.1093/cercor/bhg133

Cabeza, R., Grady, C., Nyberg, L., McIntosh, A., Tulving, E., Kapur, S., et al. (1997). Age-related differences in neural activity during memory encoding and retrieval: a position emission tomography study. J. Neurosci. 17, 391-400. doi: 10.1523/jneurosci.17-01-00391.1997

Carp, J., Gmeindl, L., and Reuter-Lorenz, P. A. (2010). Age differences in the neural representation of working memory revealed by multi-voxel pattern analysis. Front. Hum. Neurosci. 4:217. doi: 10.3389/fnhum.2010.00217

Charness, N. (2008). Aging and human performance. Hum. Fact. 50, 548-555.

Cohen, J., Cohen, P., West, S. G., and Aiken, L. S. (2003). Applied Multiple Regression/Correlation Analysis for the Behavioral Sciences, 3rd Edn. Mahwah, NJ: Erlbaum.

Cox, R. (1996). AFNI: software for analysis and visualization of functional magnetic resonance neuroimages. Comput. Biomed. Res. 29, 162-173. doi: 10.1006/cbmr. 1996.0014

Davis, S. W., Dennis, N. A., Daselaar, S. M., Fleck, M. S., and Cabeza, R. (2008). Que' PASA? The posterior-anterior shift in aging. Cereb. Cortex 18, 1201-1209. doi: $10.1093 /$ cercor/bhm155

Davis, S. W., Kragel, J. E., Madden, D. J., and Cabeza, R. (2011). The architecture of cross- hemispheric communication in the aging brain: Linking behavior to functional, and structural connectivity. Cereb. Cortex. 22, 232-242. doi: 10. 1093/cercor/bhr123

Deblaere, K., Boon, P. A., Vandemaele, P., Tieleman, A., Vonck, K., and Vingerhoets, G. (2004). MRI language dominance assessment in epilepsy patients at 1.0 T: Region of interest analysis and comparison with intracarotid amytal testing. Neuroradiology 46, 413-420.

Duda, B., Puente, A. N., and Miller, L. S. (2014). Cognitive reserve moderates relation between global cognition and functional status in older adults. J. Clin. Exp. Neuropsychol. 36, 368-378. doi: 10.1080/13803395.2014.892916

Duverne, S., Habibi, A., and Rugg, M. D. (2008). Regional specificity of age effects on the neural correlates of episodic retrieval. Neurobiol. Aging 29, 1902-1916. doi: 10.1016/j.neurobiolaging.2007.04.022

Fox, M. D., Snyder, A. Z., Vincent, J. L., Corbetta, M., Van Essen, D. C., and Raichle, M. E. (2005). The human brain is intrinsically organized into dynamic, anticorrelated functional networks. Proc. Natl. Acad. Sci. U.S.A. 102, 9673-9678. doi: 10.1073/pnas.0504136102

Gilbert, S., Bird, G., Frith, C., and Burgess, P. (2012). Does "task difficulty" explain “task-induced deactivation?”. Front. Psychol. 3:125. doi: 10.3389/fpsyg.2012. 00125

Goh, J. O. (2011). Functional dedifferentiation and altered connectivity in older adults: Neural accounts of cognitive aging. Aging Dis. 2:30.

Grady, C. (2012). The cognitive neuroscience of ageing. Nat. Rev. Neurosci. 13, 491-505. doi: 10.1038/nrn3256 
Grady, C. L. (2008). Cognitive neuroscience of aging. Ann. N.Y. Acad. Sci. 1124, 127-144.

Grady, C. L., McIntosh, A. R., and Craik, F. I. (2003). Age-related differences in the functional connectivity of the hippocampus during memory encoding. Hippocampus 13, 572-586. doi: 10.1002/hipo.10114

Grady, C. L., Springer, M. V., Hongwanishkul, D., McIntosh, A. R., and Winocur, G. (2006). Age-related changes in brain activity across the adult lifespan. J. Cogn. Neurosci. 18, 227-241. doi: 10.1162/jocn.2006.18.2.227

Greenwood, P. M. (2007). Functional plasticity in cognitive aging: Review and hypothesis. Neuropsychology 21, 657-673. doi: 10.1037/0894-4105.21.6.657

Greicius, M. D., Srivastava, G., Reiss, A. L., and Menon, V. (2004). Default-mode network activity distinguishes Alzheimer's disease from healthy aging: Evidence from functional MRI. Proc. Natl. Acad. Sci. 101, 4637-4642. doi: 10.1073/pnas. 0308627101

Gutchess, A. H., Welsh, R. C., Hedden, T., Bangert, A., Minear, M., Liu, L. L., et al. (2005). Aging and the neural correlates of successful picture encoding: frontal activations for decreased medial temporal activity. J. Cogn. Neurosci. 17:96.

Haley, A. P., Hoth, K. F., Gunstad, J., Paul, R. H., Jefferson, A. L., Tate, D. F., et al. (2009). Subjective cognitive complaints relate to white matter hyperintensities and future cognitive decline in patients with cardiovascular disease. Am. J. Geriatr. Psychiatry 17, 976-985. doi: 10.1097/JGP.0b013e3181b208ef

Hampson, M., Driesen, N. R., Skudlarski, P., Gore, J. C., and Constable, R. T. (2006). Brain connectivity related to working memory performance. J. Neurosci. 26, 13338-13343. doi: 10.1523/jneurosci.3408-06.2006

Hansen, N. L., Lauritzen, M., Mortensen, E. L., Osler, M., Avlund, K., Fagerlund, B., et al. (2014). Subclinical cognitive decline in middle-age is associated with reduced task- induced deactivation of the brain's default mode network. Hum. Brain Mapp. 35, 4488-4498. doi: 10.1002/hbm.22489

Haug, H., and Eggers, R. (1991). Morphometry of the human cortex cerebri and corpus striatum during aging. Neurobiol. Aging 12, 336-338. doi: 10.1016/ 0197-4580(91)90013-a

Hayes, A. F. (2012). PROCESS: A Versatile Computational Tool for Observed Variable Mediation, Moderation, and Conditional Process Modeling. Available at: http://www.afhayes.com/public/process2012.pdf (accessed January 17, 2017).

Hayes, A. F. (2013). Introduction to Mediation, Moderation, and Conditional Process Analysis: A Regression-Based Approach. New York, NY: Guilford Press.

Hsieh, S., and Fang, W. (2012). Elderly adults through compensatory responses can be just as capable as young adults in inhibiting the flanker influence. Biol. Psychol. 90, 113-126. doi: 10.1016/j.biopsycho.2012.03.006

Johnson, M. K., Mitchell, K. J., Raye, C. L., and Greene, E. J. (2004). An age-related deficit in prefrontal cortical function associated with refreshing information. Psychol. Sci. 15, 127-132. doi: 10.1111/j.0963-7214.2004.01502009.x

Jonides, J., Schumacher, E. H., Smith, E. E., Lauber, E. J., Awh, E., Minoshima, S., et al. (1997). Verbal working memory load affects regional brain activation as measured by PET. J. Cogn. Neurosci. 9, 462-475. doi: 10.1162/jocn.1997.9.4.462

Karbach, J., and Verhaeghen, P. (2014). Making working memory work: a metaanalysis of executive control and working memory training in older adults. Psychol. Sci. 25, 2027-2037. doi: 10.1177/0956797614548725

Katzman, R. (1993). Education and the prevalence of dementia and Alzheimer's disease. Neurology 43, 13-20.

Klaassens, B. L., van Gerven, J. M., van der Grond, J., de Vos, F., Möller, C., and Rombouts, S. A. (2017). Diminished posterior precuneus connectivity with the default mode network differentiates normal aging from Alzheimer's disease. Front. Aging Neurosci. 9:97. doi: 10.3389/fnagi.2017.00097

Kray, J., Li, K. Z., and Lindenberger, U. (2002). Age-related changes in taskswitching components: the role of task uncertainty. Brain Cogn. 49, 363-381. doi: 10.1006/brcg.2001.1505

Kray, J., and Lindenberger, U. (2000). Adult age differences in task switching. Psychol. Aging 15:126. doi: 10.1037//0882-7974.15.1.126

Lee, Y., Grady, C. L., Habak, C., Wilson, H. R., and Moscovitch, M. (2011). Face processing changes in normal aging revealed by fMRI adaptation. J. Cogn. Neurosci. 23, 3433-3447. doi: 10.1162/jocn_a_00026

Li, S. C., and Lindenberger, U. (1999). "Cross-level unification: A computational exploration of the link between deterioration of neurotransmitter systems and dedifferentiation of cognitive abilities in old age," in Cognitive Neuroscience of Memory, eds L.-G. Nilsson and H. J. Markowitsch (Ashland, OH: Hogrefe \& Huber), 103-146.
Li, S. C., and Sikström, S. (2002). Integrative neurocomputational perspectives on cognitive aging, neuromodulation, and representation. Neurosci. Biobehav. Rev. 26, 795-808. doi: 10.1016/s0149-7634(02)00066-0

Li, Z., Moore, A. B., Tyner, C., and Hu, X. (2009). Asymmetric connectivity reduction and its relationship to "HAROLD" in aging brain. Brain Res. 1295, 149-158. doi: 10.1016/j.brainres.2009.08.004

Logan, J. M., Sanders, A. L., Snyder, A. Z., Morris, J. C., and Buckner, R. L. (2002). Under- recruitment and nonselective recruitment: dissociable neural mechanisms associated with aging. Neuron 33, 827-840. doi: 10.1016/s08966273(02)00612-8

Lustig, C., Snyder, A. Z., Bhakta, M., O’Brien, K. C., McAvoy, M., Raichle, M. E., et al. (2003). Functional deactivations: change with age and dementia of the Alzheimer type. Proc. Natl. Acad. Sci. 100, 14504-14509. doi: 10.1073/pnas. 2235925100

Madden, D. J. (1990). Adult age differences in attentional selectivity and capacity. Eur. J. Cogn. Psychol. 2, 229-252. doi: 10.1080/09541449008406206

Madden, D. J., Gottlob, L. R., Denny, L. L., Turkington, T. G., Provenzale, J. M., Hawk, T. C., et al. (1999a). Aging and recognition memory: changes in regional cerebral blood flow associated with components of reaction time distributions. J. Cogn. Neurosci. 11, 511-520. doi: 10.1162/089892999563571

Madden, D. J., Turkington, T. G., Provenzale, J. M., Denny, L. L., Hawk, T. C., Gottlob, L. R., et al. (1999b). Adult age differences in the functional neuroanatomy of verbal recognition memory. Hum. Brain Mapp. 7, 115-135. doi: 10.1002/(sici) 1097-0193(1999)7:2<115::aid-hbm5>3.0.co;2-n

Mattay, V. S., Fera, F., Tessitore, M. D., Hariri, A. R., Das, S., Callicott, J. H., et al. (2002). Neurophysiological correlates of age-related changes in human motor function. Neurology 58, 630-635. doi: 10.1212/wnl.58.4.630

McDonough, I. M., Wong, J. T., and Gallo, D. A. (2012). Age-related differences in prefrontal cortex activity during retrieval monitoring: testing the compensation and dysfunction accounts. Cereb. Cortex 23, 1049-1060. doi: 10.1093/cercor/ bhs 064

McIntosh, A. R., Sekuler, A. B., Penpeci, C., Rajah, M. N., Grady, C. L., Sekuler, R., et al. (1999). Recruitment of unique neural systems to support visual memory in normal aging. Curr. Biol. 9, 1275-1278.

Milham, M. P., Erickson, K. I., Banich, M. T., Kramer, A. F., Webb, A., Wszalek, T., et al. (2002). Attentional control in the aging brain: insights from an fMRI study of the stroop task. Brain Cogn. 49, 277-296. doi: 10.1006/brcg.2001.1501

Miller, S. L., Celone, K., DePeau, K., Diamond, E., Dickerson, B. C., Rentz, D. et al. (2008). Age-related memory impairment associated with loss of parietal deactivation but preserved hippocampal activation. Proc. Natl. Acad. Sci. 105, 2181-2186. doi: 10.1073/pnas.0706818105

Morcom, A. M., and Friston, K. J. (2012). Decoding episodic memory in ageing: a Bayesian analysis of activity patterns predicting memory. Neuroimage 59, 1772-1782. doi: 10.1016/j.neuroimage.2011.08.071

National Institute on Aging and World Health Organization (2011). Global Health and Aging. Available at: http://www.nia.nih.gov/research/publication/globalhealth-and aging/humanity's-aging (accessed April 19, 2018).

Ng, K. K., Lo, J. C., Lim, J. K., Chee, M. W., and Zhou, J. (2016). Reduced functional segregation between the default mode network and the executive control network in healthy older adults: a longitudinal study. NeuroImage 133, 321-330. doi: 10.1016/j.neuroimage.2016.03.029

Owen, A. M., McMillan, K. M., Laird, A. R., and Bullmore, E. (2005). NBack working memory paradigm: a meta-analysis of normative functional neuroimaging studies. Hum. Brain Mapp. 25, 46-59. doi: 10.1002/hbm. 20131

Park, D. C., Polk, T. A., Hebrank, A. C., and Jenkins, L. (2010). Age differences in default mode activity on easy and difficult spatial judgment tasks. Front. Hum. Neurosci. 3:75. doi: 10.3389/neuro.09.075.2009

Park, D. C., Polk, T. A., Park, R., Minear, M., Savage, A., and Smith, M. R. (2004). Aging reduces neural specialization in ventral visual cortex. Proc. Natl. Acad. Sci. 101, 13091-13095. doi: 10.1073/pnas.0405148101

Park, D. C., and Reuter-Lorenz, P. (2009). The adaptive brain: aging and neurocognitive scaffolding. Ann. Rev. Psychol. 60, 173-196. doi: 10.1146/ annurev.psych.59.103006.093656

Parker, S., Jagger, C., Lamura, G., Chiatti, C., Wahl, H., Iwarsson, S., et al. (2012). FUTURAGE: creating a roadmap for ageing research. Eur. Geriatr. Med. 3, S100-S101. 
Persson, J., Lustig, C., Nelson, J. K., and Reuter-Lorenz, P. A. (2007). Age differences in deactivation: a link to cognitive control? J. Cogn. Neurosci. 19, 1021-1032. doi: 10.1162/jocn.2007.19.6.1021

Persson, J., Nyberg, L., Lind, J., Larsson, A., Nilsson, L.-G., Ingvar, M., et al. (2005). Structure-function correlates of cognitive decline in aging. Cereb. Cortex 16, 907-915. doi: 10.1093/cercor/bhj036

Persson, J., Sylvester, C. Y., Nelson, J. K., Welsh, K. M., Jonides, J., and ReuterLorenz, P. A. (2004). Selection requirements during verb generation: differential recruitment in older and younger adults. Neuroimage 23, 1382-1390. doi: 10.1016/j.neuroimage.2004.08.004

Petrella, J. R., Sheldon, F. C., Prince, S. E., Calhoun, V. D., and Doraiswamy, P. M. (2011). Default mode network connectivity in stable vs progressive mild cognitive impairment. Neurology 76, 511-517. doi: 10.1212/WNL. 0b013e31820af94e

Rajah, M. N., and D'Esposito, M. (2005). Region-specific changes in prefrontal function with age: a review of PET and PMRI studies on working and episodic memory. Brain 128, 1964-1983. doi: 10.1093/brain/awh608

Raz, N., Rodrigue, K. M., Head, D., Kennedy, K. M., and Acker, J. D. (2004). Differential aging of the medial temporal lobe: a study of a five-year change. Neurology 62, 433-438. doi: 10.1212/01.wnl.0000106466.09835.46

Reuter-Lorenz, P. A., and Cappell, K. A. (2008). Neurocognitive aging and the compensation hypothesis. Curr. Dir. Psychol. Sci. 17, 177-182. doi: 10.1111/j. 1467-8721.2008.00570.x

Reuter-Lorenz, P. A., Jonides, J., Smith, E. E., Hartley, A., Miller, A., Marshuetz, C., et al. (2000). Age differences in the frontal lateralization of verbal and spatial working memory revealed by PET. J. Cogn. Neurosci. 12, 174-187. doi: 10.1162/089892900561814

Rombouts, S. A., Barkhof, F., Goekoop, R., Stam, C. J., and Scheltens, P. (2005). Altered resting state networks in mild cognitive impairment and mild Alzheimer's disease: an fMRI study. Hum. Brain Mapp. 26, 231-239. doi: 10. 1002/hbm. 20160

Rossi, S., Miniussi, C., Pasqualetti, P., Babiloni, C., Rossini, P. M., and Cappa, S. F. (2004). Age-related functional changes in prefrontal cortex and long-term memory: a repetitive transcranial magnetic stimulation study. J. Neurosci. 24, 7939-7944. doi: 10.1523/jneurosci.0703-04.2004

Rypma, B., Eldreth, D. A., and Rebbechi, D. (2007). Age-related differences in activation- performance relations in delayed-response tasks: a multiple component analysis. Cortex 43, 65-76. doi: 10.1016/s0010-9452(08) 70446-5

Salthouse, T. A. (1996). The processing-speed theory of adult age differences in cognition. Psychol. Rev. 103, 403-428. doi: 10.1037//0033-295x.103.3.403

Sebastian, A., Baldermann, C., Feige, B., Katzev, M., Scheller, E., Hellwig, B., et al. (2013). Differential effects of age on subcomponents of response inhibition. Neurobiol. Aging 34, 2183-2193. doi: 10.1016/j.neurobiolaging.2013.03.013

Smith, E. E., and Jonides, J. (1999). Storage and executive processes in the frontal lobes. Science 283, 1657-1661. doi: 10.1126/science.283.5408.1657

Spreng, R. N. (2012). The fallacy of a "task-negative" network. Front. Psychol. 3:145. doi: 10.3389/fpsyg.2012.00145
Spreng, R. N., Stevens, W. D., Viviano, J. D., and Schacter, D. L. (2016). Attenuated anticorrelation between the default and dorsal attention networks with aging: evidence from task and rest. Neurobiol. Aging 45, 149-160. doi: 10.1016/j. neurobiolaging.2016.05.020

Stern, Y. (2012). Cognitive reserve in ageing and Alzheimer's disease. Lancet Neurol. 29, 997-1003. doi: 10.1016/j.biotechadv.2011.08.021

Stern, Y. (2016). An approach to studying the neural correlates of reserve. Brain Imag. Behav. 11, 410-416. doi: 10.1007/s11682-016-9566-x

Suchy, Y., Kraybill, M. L., and Franchow, E. (2011). Instrumental activities of daily living among community-dwelling older adults: discrepancies between self-report and performance are mediated by cognitive reserve. J. Clin. Exp. Neuropsychol. 33, 92-100. doi: 10.1080/13803395.2010.493148

Sweet, L. H., Paskavitz, J. F., Haley, A. P., Gunstad, J. J., Mulligan, R. C., Nyalakanti, P. K., et al. (2008). Imaging phonological similarity effects on verbal working memory. Neuropsychologia 46, 1114-1123. doi: 10.1016/j.neuropsychologia. 2007.10.022

Thomsen, T., Specht, K., Hammar, A., Nyttingnes, J., Ersland, L., and Hugdahl, K. (2004). Brain localization of attention control in different age groups by combining functional and structural MRI. Neuroimage 22, 912-919. doi: 10. 1016/j.neuroimage.2004.02.015

Turner, G. R., and Spreng, R. N. (2015). Prefrontal engagement and reduced default network suppression co-occur and are dynamically coupled in older adults: the default-executive coupling hypothesis of aging. J. Cogn. Neurosci. 27, 2462-2476. doi: 10.1162/jocn_a_00869

Valenzuela, M. J., Sachdev, P., Wen, W., Chen, X., and Brodaty, H. (2008). Lifespan mental activity predicts diminished rate of hippocampal atrophy. PLoS One 3:e2598. doi: 10.1371/journal.pone.0002598

Yuan, W., Szaflarski, J. P., Schmithorst, V. J., Schapiro, M., Byars, A. W., and Strawsburg, R. H. (2006). FMRI shows atypical language lateralization in pediatric epilepsy patients. Epilepsia 47, 593-600. doi: 10.1111/j.1528-1167. 2006.00474.x

Zacks, R. T., Hasher, L., and Li, K. Z. (2000). "Human memory," in Handbook of Aging and Cognition, 2nd Edn, eds T. A. Salthouse and F. I. M. Craik (Mahwah, NJ: Lawrence Erlbaum), 293-357.

Zarahn, E., Rakitin, B., Abela, D., Flynn, J., and Stern, Y. (2007). Age-related changes in brain activation during a delayed item recognition task. Neurobiol. Aging 28, 784-798. doi: 10.1016/j.neurobiolaging.2006.03.002

Conflict of Interest Statement: The authors declare that the research was conducted in the absence of any commercial or financial relationships that could be construed as a potential conflict of interest.

Copyright (c) 2019 Duda, Owens, Hallowell and Sweet. This is an open-access article distributed under the terms of the Creative Commons Attribution License (CC BY). The use, distribution or reproduction in other forums is permitted, provided the original author(s) and the copyright owner(s) are credited and that the original publication in this journal is cited, in accordance with accepted academic practice. No use, distribution or reproduction is permitted which does not comply with these terms. 